\title{
DOPPLER IMAGING OF V404 CYG IN QUIESCENCE
}

\author{
JORGE CASARES \\ University of Oxford, Department of Physics, Nuclear Physics \\ Laboratory, Keble Road, Oxford OX1 3RH, UK
}

\begin{abstract}
We present Doppler images of the accretion disc in the black hole X-ray transient V404 Cyg during its quiescent phase. The $\mathrm{H} \alpha$ map shows evidence for a hot spot, indicating that mass transfer is continuing long after the 1989 outburst. Both the $\mathrm{H} \alpha$ and the IR CaIl emission maps exhibit intense bright spots at the position of the secondary star, which we take as a sign of chromospheric activity. The CaII emission arises from the outer parts of the accretion disc, and its surface brightness distribution is consistent with dwarf novae discs $\left(I \propto R^{-3 / 2}\right)$.
\end{abstract}

\section{Introduction}

V404 Cyg is the optical counterpart of the X-ray transient GS 2023+338, which was discovered by Ginga at the peak of its recent 1989 outburst [6]. Its striking X-ray behaviour received great attention, leading Tanaka [13] to propose V404 Cyg as a new black hole candidate. These include a hard power-law spectrum (without an ultra-soft component) with associated millisecond flickering, chaotic variability (varying by a factor of 200 in a few minutes) and a luminosity saturation effect at $L_{\mathrm{x}} \simeq 1.510^{38}(\mathrm{~d} / \mathrm{kpc})^{2} \mathrm{erg} \mathrm{s}^{-1}$.

Since 1990 the system has settled down in quiescence at $V \sim 18.5$ mag. Follow-up spectroscopy led to the discovery of late-type absorption features moving at $211 \mathrm{~km} \mathrm{~s}^{-1}$ in a $6.5 \mathrm{~d}$ orbit. With a mass function of $f(M)=6.3 \pm 0.3 \mathrm{M}_{\odot}, \mathrm{V} 404 \mathrm{Cyg}$ provides the strongest dynamical evidence yet for a black hole [3]. The spectrum of the K0 secondary also showed the puzzling presence of the ${ }^{7} \mathrm{Li}$ resonance line [9], suggesting perhaps the action of spallation reactions during the outbursts (see [10]). V404 Cyg is also remarkable because of the exhibition of a strong $6 \mathrm{~h}$ variability in the continuum and emission lines $[1,4]$, the origin of which remains unknown.

A. Evans and J. H. Wood (eds.), Cataclysmic Variables and Related Objects, 395-398. (C) 1996 Kluwer Academic Publishers. Printed in the Netherlands. 


\section{Database and updated system parameters}

With a period of $6.5 \mathrm{~d}$ it is difficult to sample a full cycle of V404 Cyg adequately within a single campaign. Therefore, we have attempted to monitor the source continuously since the start of the quiescent phase. Our database now consists of $73 \mathrm{H} \alpha$ spectra from $1990-91\left(0.75 \AA\right.$ pixel $\left.^{-1}\right)$ and 84 spectra from $1992-94\left(0.39 \AA\right.$ pixel $\left.^{-1}\right)$. In addition, during 1992 and 1994 we also acquired 58 spectra in the region of the IR CaII triplet, at a dispersion of $0.75 \AA$ pixel $^{-1}$. In Table 1 we summarize the updated system parameters and refined rotational broadening $(V \sin i)$ as derived by using the K0IV template star HR8857 in both wavelength ranges independently (further details of the process can be found in [2]). Note the excellent agreement between all the quantities. As a matter of precaution, we initially avoided the $\mathrm{Ca}$ II triplet lines in the cross-correlation analysis of the IR spectra (there is evidence for broad Ca II emission). However, a further test using these lines alone has provided identical orbital parameters.

TABLE 1. System parameters

\begin{tabular}{lcccc}
\hline$\lambda$-range & $\gamma\left(\mathrm{km} \mathrm{s}^{-1}\right)$ & Period $(\mathrm{d})$ & $\mathrm{K}\left(\mathrm{km} \mathrm{s}^{-1}\right)$ & $V \sin i\left(\mathrm{~km} \mathrm{~s}^{-1}\right)$ \\
\hline $\mathrm{H} \alpha$ & $0.3 \pm 0.6$ & $6.47129 \pm 710^{-5}$ & $208.4 \pm 0.6$ & $40.8^{\dagger} \pm 0.9$ \\
$\mathrm{Ca} \mathrm{II}$ & $0.2 \pm 0.8$ & $6.47129 \pm 810^{-5}$ & $207.6 \pm 1.1$ & $41.1 \pm 1.0$ \\
\hline
\end{tabular}

$\dagger$ The slight difference with [2] is due to a small bug found in the broadening routine.

Our revised values for the mass function and mass ratio are $f(M)=$ $6.07 \pm 0.05 \mathrm{M}_{\odot}$ and $q=M_{\mathrm{c}} / M_{\mathrm{x}}=0.067 \pm 0.005$ respectively. Combining these with the limits to the inclination $\left(i=52^{\circ} \ldots 60^{\circ}[12]\right)$ brings the black hole mass to the range $10.5 \ldots 14 \mathrm{M}_{\odot}$.

In order to study the line emission regions in V404 Cyg we first need to correct for the contamination by the spectrum of the secondary star. This was performed by subtracting our HR8857 spectrum (broadened by $41 \mathrm{~km} \mathrm{~s}^{-1}$ and shifted to the secondary's frame) from each individual spectrum of V404 Cyg. The appropriate fraction to be subtracted was estimated following the prescription of Marsh et al. [8]. An outcome of this analysis is a possible dimming of the accretion disc contribution with time, particularly from 1991 to 1992 . Therefore, we decided to exclude the lower resolution spectra (1990-1991) from our subsequent analysis. The spectral averages, with the secondary star removed, are presented in Fig. 1. The Ca II triplet lines appear to be saturated (all have $\mathrm{EW} \simeq 20 \AA$ ) and hence they are optically thick, in contrast to the Balmer emission [4]. 

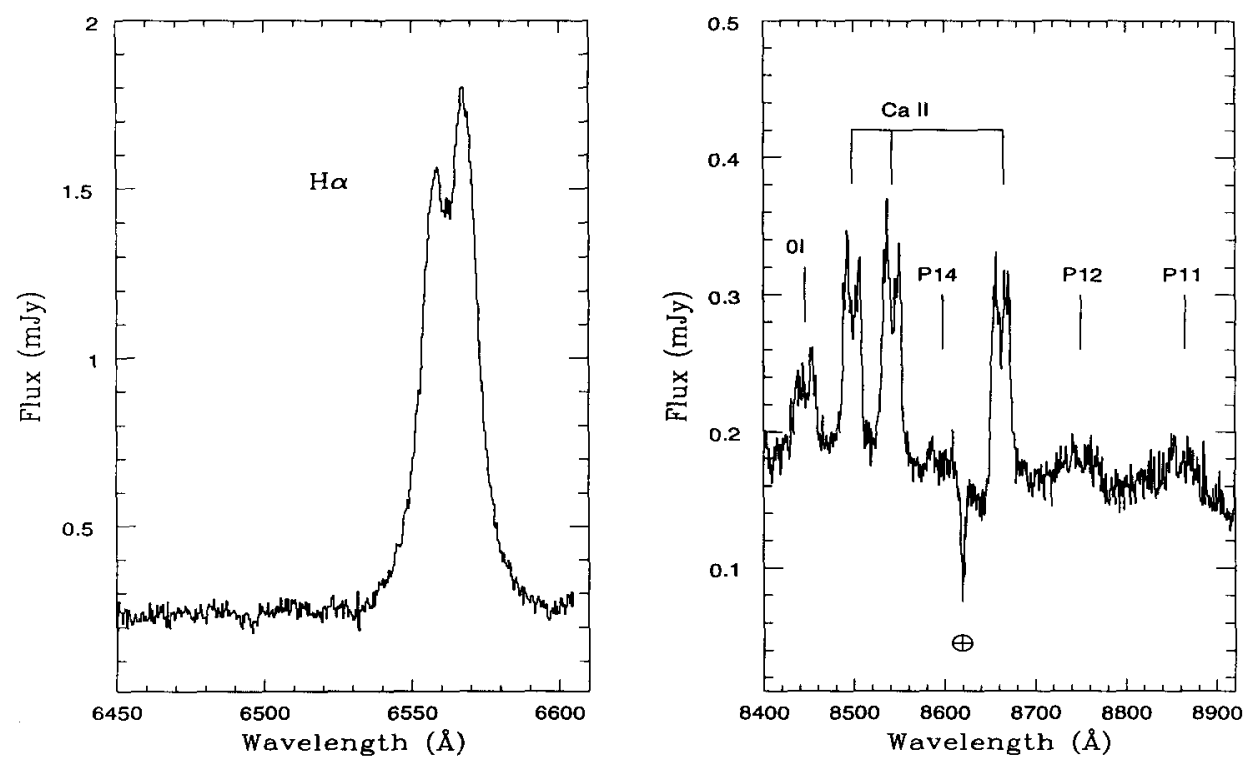

Figure 1. Averaged spectra after subtracting the contribution of the companion star.

\section{Doppler imaging}

Images of the $\mathrm{H} \alpha$ and CaII emission regions in V404 Cyg have been constructed through combining the phase-resolved profiles using the Doppler tomography technique [7]. These are presented in Fig. 2. The $\mathrm{H} \alpha$ map shows extended emission close to where the gas stream impacts the outer edge of the accretion disc. This implies that mass transfer proceeds during quiescence and permits us to constrain the outer disc radius to $\sim 0.6 R_{\mathrm{L} 1}$. Both the $\mathrm{H} \alpha$ and the CaII maps exhibit bright spots at the position of the secondary. Although some of this might be a residual of the secondary subtraction (see [8]), we associate such emission with chromospheric activity, as is frequently observed in rapid rotators (e.g. RS CVn stars [11]). The possibility that some emission is powered by X-ray heating of the inner hemisphere is disproved by the negligible ellipticity of the radial velocity curve of the Ca II absorption lines.

The emission-line surface brightness $(I)$ of the accretion disc can be extracted from the Doppler images after assuming that the velocity field is Keplerian. The disc surface brightness peaks further away for the CaII lines than for $\mathrm{H} \alpha$. In addition, $I_{\mathrm{Ca} \text { II }} \propto R^{-1.4}$, consistent with dwarf novae discs [5], whereas a less steep behaviour is found for $\mathrm{H} \alpha\left(I_{\mathrm{H} \alpha} \propto R^{-0.7}\right)$. 


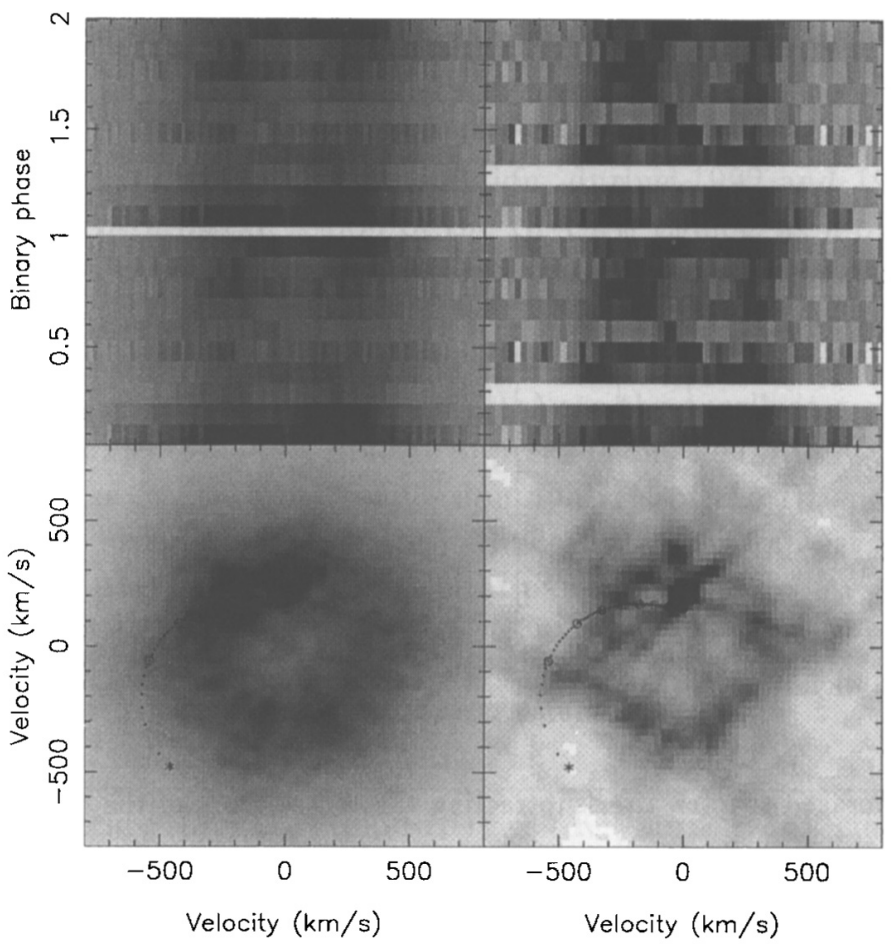

Figure 2. Trailed spectra and Doppler maps of $\mathrm{H} \alpha$ and $\mathrm{Ca}$ II $\lambda 8542$.

\section{References}

1. Casares, J., Charles, P.A., 1992, MNRAS, 255, 7

2. Casares, J., Charles, P.A., 1994, MNRAS, 271, 5p

3. Casares, J., Charles, P.A., Naylor, T., 1992, Nature, 355, 614

4. Casares, J., Charles, P.A., Naylor, T., Pavlenko, E.P., 1993, MNRAS, 265, 834

5. Horne, K., Saar, S.H., 1991, Ap. J., 374, L55

6. Marsden, B.G., 1989, IAUC 4783

7. Marsh, T.R., Horne, K., 1988, MNRAS, 235, 269

8. Marsh, T.R., Robinson, E.L., Wood, J.H., 1994 MNRAS, 266, 137

9. Martín, E. L., Rebolo, R., Casares, J., Charles, P.A., 1992, Nature, 358, 129

10. Martín, E. L., Rebolo, R., Casares, J., Charles, P.A., 1994, Ap. J., 435, 791

11. Rutten, R.G.M., 1987, A\&A, 177, 131

12. Shahbaz, T., Ringwald, F.A., Bunn, J.C., et al., 1994, MNRAS, 271, 10p

13. Tanaka, Y., 1989, in "Proc. 23rd ESLAB Symp. on Two-Topics in X-Ray Astronomy", ed. J. Hunt \& B. Battrick (ESA Publ. Division), p3 